\title{
Respiratory Motion Analysis: Towards Gated Augmentation of the Liver
}

\author{
B. Olbrich ${ }^{\mathrm{a}}$, J. Traub ${ }^{\mathrm{b}}{ }^{*}$, S. Wiesner ${ }^{\mathrm{b}}$, A. Wichert ${ }^{\mathrm{a}}$, \\ H. Feussner ${ }^{\mathrm{a}}$, N. Navab \\ ${ }^{a}$ Institut für Minimal-invasive therapeutische Interventionen (MITI), Klinikum Rechts der Isar, \\ Technische Universität München \\ ${ }^{b}$ Chair for Computer Aided Medical Procedures(CAMP), Fakultät für Informatik, \\ Technische Universität München
}

\begin{abstract}
Laparoscopic surgery had a rapid development over the past decade. However, the view of the surgeon is limited to the image of the laparoscope. Augmented reality can provide further information to the surgeon by enabling a view inside the patient, and thus supporting a more precise and less invasive procedure.

The limiting factors for realistic augmentation during liver surgery are movement and deformation of the organ due to respiratory motion. In our experiments we analyzed respiratory motion patterns of the liver caused by the respirator. Throughout our experiments we validated our assumption that repositioning after one breathing cycle is within a range of $1 \mathrm{~mm}$. For an optimal augmentation of the liver in the laparoscopic image we suggest to adjust the respirator thus that we have a static exhalation phase of two to three seconds on which our augmentation is performed.
\end{abstract}

Keywords: Liver Motion; Augmented Reality; Gated Augmentation; Respiratory Motion

\section{Introduction}

Augmented reality is a promising technology for enhancement of minimally invasive surgery, where the view of the region of interest is limited to the endoscopic/laparoscopic view [1,2,3]. In particular, the use of augmented reality for laparoscopic liver surgery could provide higher accuracy, better performance and eventually a decrease in operating time. The main idea is to guide the surgeon to the desired target region by an overlay of medical imaging, such as CT or MR, and/or planning data onto the real laparoscopic view.

Recent advances in augmented reality technology have led to promising solutions in terms of robustness, speed and accuracy for overlaying (virtual) medical data on the real view of non deforming patient's anatomy [4,5]. A major obstacle for accurate augmentation in laparoscopic surgery is the organ deformation due to respiratory motion [6]. However, intraoperatively the patient is narcotized and the breathing is controlled by the respirator. We have analyzed the correlation between the configurations (input/output parameters) of the respirator and liver motion. We show that we can use this correlation to augment the laparoscopic image only in well defined stages of the respiratory cycle.

\footnotetext{
*traub@cs.tum.edu
} 


\section{Purpose}

The main idea is to reduce the perturbation of the augmentation by respiratory motion by augmenting the laparoscopic view at a given respiratory state. Current CT technology allows us to acquire data during patient's breath hold. In this case, intraoperatively the augmentation would occur only during the corresponding moment within the respiratory cycle. This could allow us to provide useful intraoperative augmentation results without real-time deformable registration of preoperative CT to intraoperative optical or US data. Beside laparoscopic surgery other potential applications of such respiratory gated augmentation include the guidance for needle biopsy and RF ablation of the liver [7].

\section{Methods}

The analysis of the correlation between the respirator and liver motion was performed on 15 pigs. We attached three sensors of an electromagnetic tracking device (MiniBIRD 800 from Ascension) to specific points on the surface of the liver. The sensors are extended by a customized frame and sutured onto the liver. The transmitter was well positioned to cover the entire working volume and the use of ferromagnetic material in its local neighborhood was reduced to a minimum. The acquired data was analyzed by Explorative Data Analysis to deal with the relatively high number of noise and erratic in the measurement. The first step of the Explorative Data Analysis was a standard k-means clustering to distinguish between noise outliers and liver motion. For $\mathrm{k}=7$ the cluster analysis produced the most distinct clusters. One cluster contains the relatively undisturbed liver motion data and the rest of the clusters represent the different kinds of noise and errors.

The next step of the data analysis was a qualitative analysis by means of self organizing feature maps [8]. These self organizing feature maps allow the evaluation of the quality of the single measurements as well as the evaluation of the data quality of the whole surgery. The last step of the data analysis was the evaluation of the frequency domain by applying the Fourier transformation on the spatial data.

In parallel to the data analysis, we started to implement an augmented reality system, which aims at augmenting the laparoscopic view only during the exhalation phase. The implemented software has only been applied on phantoms. The position and orientation of the laparoscopic camera (Telecam SL NTSC from Storz) is tracked by an external optical tracking system (Smarttrack2 from ART). We extended the camera by an arrangement of eight optical markers. The redundant arrangement of markers provides us with a larger working volume. For the calibration of the camera we use the Matlab Toolbox [9]. The spatial registration of the CT data from the liver phantom to the real phantom is performed by a standard 3D/3D registration algorithm [10]. The registered model is only visualized in the exhalation plateau. The results of the data analysis show that there is hardly any motion in the exhalation plateau of the different respiratory motion cycles. Therefore a periodic augmentation of liver within the exhalation plateau could provide satisfactory result. 


\section{Results}

Three sensors of the tracking device where attached to the liver of 15 different pigs during experimental surgeries. Nine different arrangements of the sensors were applied during the course of this study. A minimum of five different measurements were performed for each arrangement. We considered only measurements that were located in the first cluster of the k-means clustering and had a good qualitative representation on the self organizing feature maps. Furthermore Fourier transform of the measurement must have one distinct global maximum and it must not be correlated by significant noise. Further analysis of these valid measurements allows the detection of the predominant factor of respiratory induced liver motion. This predominant factor is the respiration frequency controlled by the respirator. Moreover the analysis of the measurements shows that other factors like heart frequency for example have minor influence and cannot be sufficiently distinguished from other noise factors. Fig. 1 shows one particular measurement. In the upper row the sensor motion in spatial domain and below the frequency domain is shown.

Considering these results, we analyzed the respiration cycle controlled by a respirator. During expiration and inspiration phase the liver motion is maximal. Our results confirm the literature $[11,12]$ that in between the expiration phase and inspiration phase of a respiratory cycle there are two plateaus where almost no liver motion is noticeable. These two plateaus are the inspiration plateau and the expiration plateau. Our measurements show that after one respiratory cycle the Euclidean distance to the corresponding measurement of another cycle has a mean difference of $0.8 \mathrm{~mm}$ with a standard deviation of $0.43 \mathrm{~mm}$.
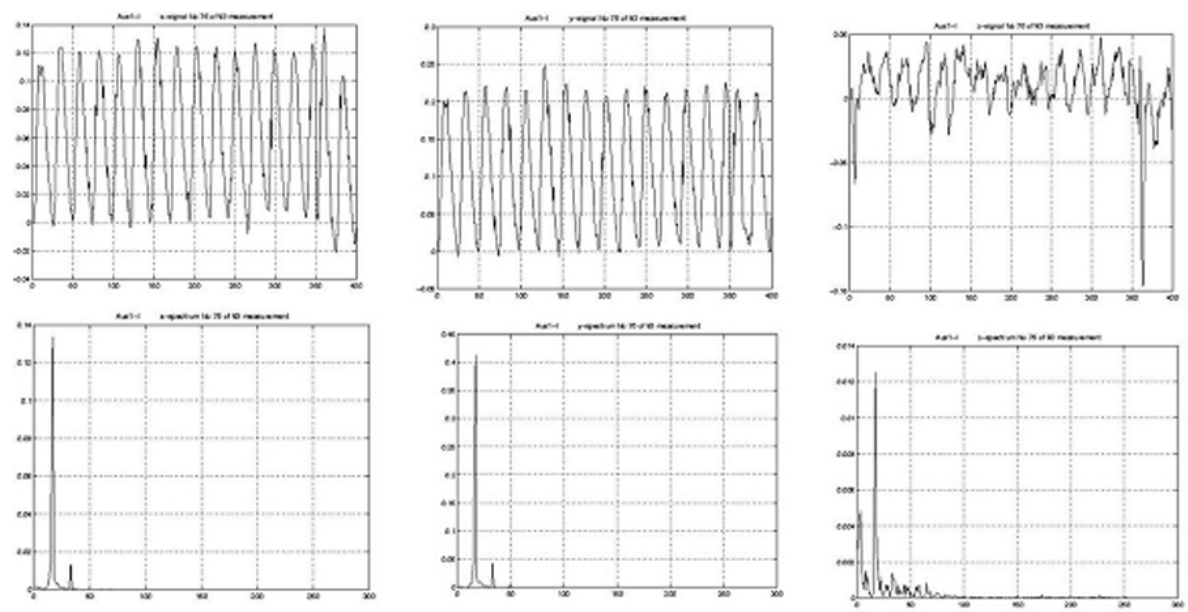

Fig. 1: The motion of one experiment in the spatial domain (upper row) and in the frequency domain (lower row). The show the $\mathrm{x}, \mathrm{y}$, and $\mathrm{z}$ directions in the tracker coordinate frame from the left to the right. 


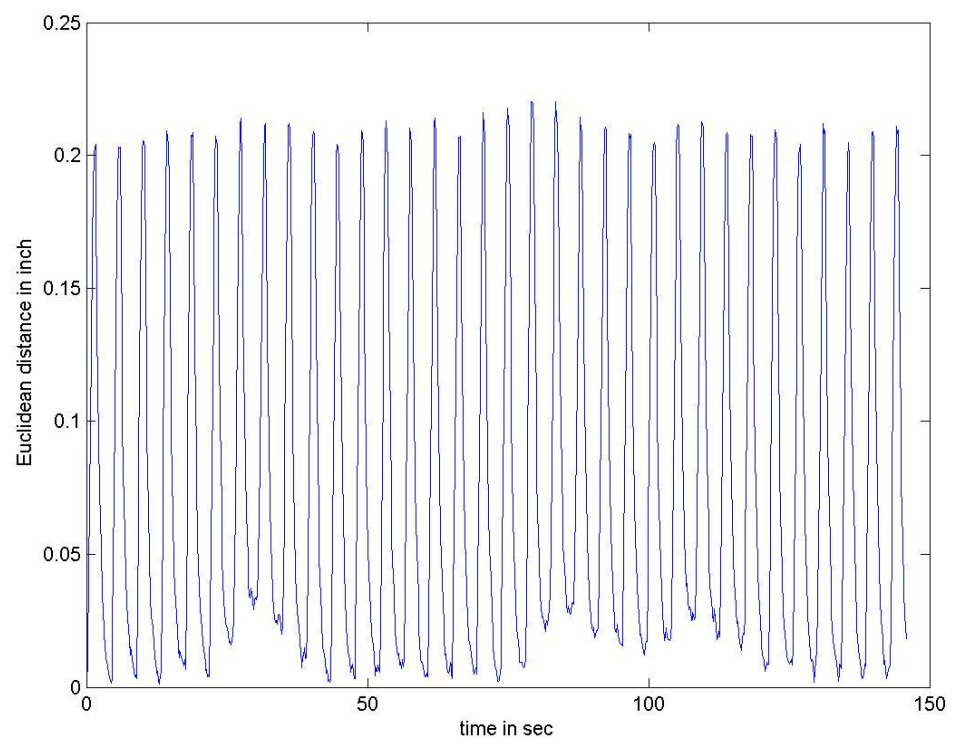

Fig. 2: Shows the Euclidean distance of each measurement to the first measurement of the exhalation plateau.

Fig. 2 shows the Euclidean distance of the measurements to the first positional measurement of the exhalation plateau. The length of the plateaus can be varied by changing parameters of the respirator. It is easier to enlarge the expiration plateau under standard respiration configuration. Therefore we used the exhalation plateau as the basic plateau phase for further applications. During this plateau there is only slight liver motion due to noise factors and information can be accurately projected by augmented reality.

The implementation of the augmented reality system shows good results in the camera calibration ( 0.3 pixel reprojection error) and reasonable results in the spatial registration $(<0.8 \mathrm{~mm}$ RMS $)$. The duration of the augmentation phase can be flexible adjusted to the duration of the exhalation plateau. However this was only tested on phantoms. We recorded a video stream of a laparoscope with the motion of the liver where the laparoscope was mounted on a steady arm. Offline we augmented this video stream with a virtual model of the liver to test the effect of the partial periodic augmentation. This showed promising results in the visualization. In Fig. 3 the first screen shows the start of the exhalation plateau and the second screen shows the end of the exhalation plateau, where there is almost no motion. The third screen shows the start of the respiratory motion. In screen number four the motion amplitude is at a maximum (inhalation plateau, very short). The fifth screen shows the beginning of the next exhalation plateau which is exactly a one cycle shift from the first screen. The augmentation of the liver is only applied in the phase between the screens one and two. 


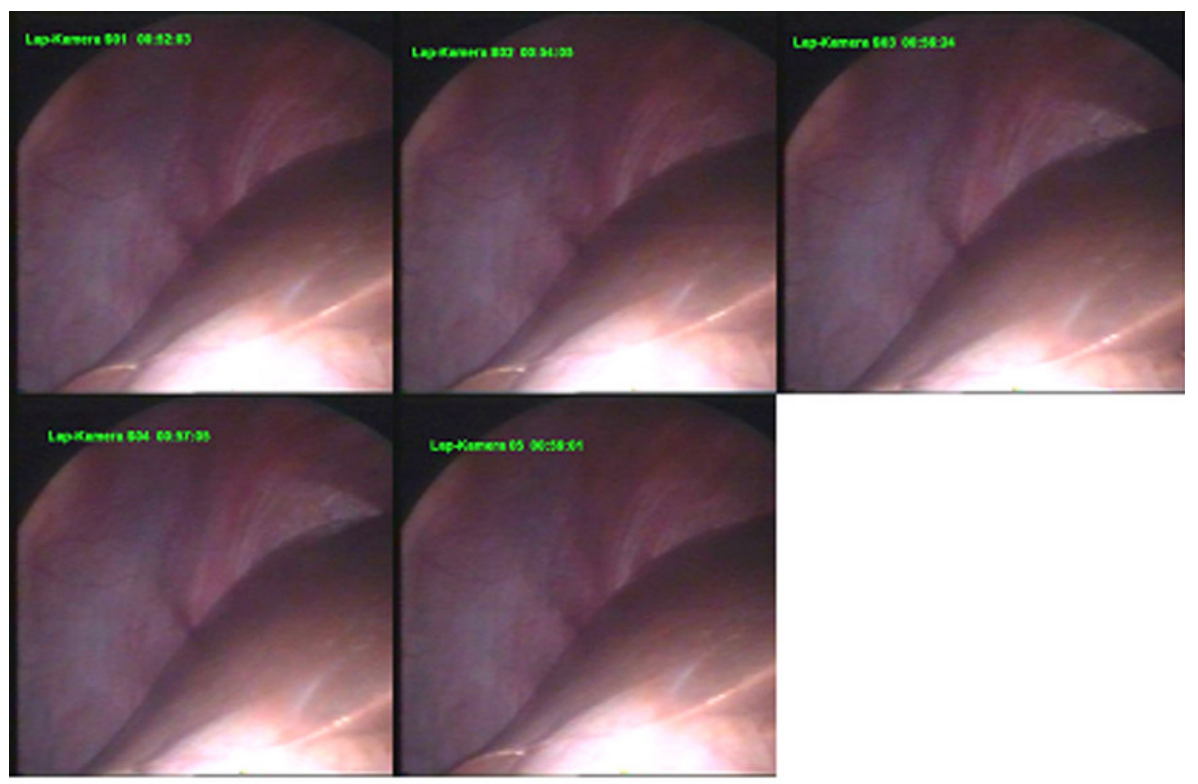

Fig. 3: Screenshot of the laparoscope mounted on a steady arm during different stages of a respiratory cycle: (a) on the exhalation plateau; (b) the inhalation phase; (c) the inhalation plateau; (d) the exhalation phase; (e) the exhalation plateau in the next phase.

\section{Conclusion}

This paper first presents an exhaustive analysis of liver motion and its correlation with controlled respiration. It then aims at real-time augmentation of laparoscopic view by preoperative CT data. It proposes a gated augmentation solution as an alternative to realtime deformable registration followed by view augmentation. First results of the motion analysis and the implementation of gated augmentation show that a partial augmentation of the scene in the exhalation phase is a promising method for safe and precise intraoperative navigation. Further experiments are required in order to prove that this partial periodic augmentation is sufficient for clinical integration. However, our first feedback was that a precise partial augmentation is more valuable than a permanent augmentation without a perfect compensation of the organ motion. Following our phantom study, we are currently working on a complete respiratory gated augmentation system. The gated augmentation will be tested and further improved if necessary. The system will then be adapted for integration into the clinical workflow. Latest results will be reported during the conference. 


\section{References}

[1] H. Fuchs, M. A. Livingston, R. Raskar D. Colucci, K. Keller, A. State, J. R. Crawford, P. Rademacher, S. H. Drake, A. A. Meyer, Augmented Reality Visualization for Laparoscopic Surgery, In Proc. Medical Image Computing and Computer Assisted Intervention (MICCAI), 1998

[2] F. Mourgues, T. Vieville, V. Falk, and E. Coste- Maniere. Interactive guidance by image overlay in robot assisted coronary artery bypass. In Proc. Medical Image Computing and Computer Assisted Intervention (MICCAI), 2003.

[3] M.Scheuering, A. Schneider, A. Schenk, B. Preim, G. Greiner. Intraoperative Augmented Reality for Minimally Invasive Liver Interventions, in Proc. SPIE Medical Imaging, San Diego (USA), 2003.

[4] F. Sauer, A. Khamene, and S. Vogt, An Augmented Reality Navigation System with a Single-Camera Tracker: System Design and Needle Biopsy Phantom Trial, in Proceedings of the Second International Conference on Medical Image Computing and Computer-Assisted Intervention (MICCAI), 2002

[5] W. Birkfellner, M. Figl, K. Huber, F. Watzinger, F. Wanschitz, J. Hummel, R. Hanel, W. Greimel, P. Homolka, R. Ewers, and H. Bergmann, A Head-Mounted Operating Binocular for Augmented Reality Visualization in Medicine-Design and Initial Evaluation, in IEEE Transaction on Medical Imaging, 21(8), 2002, pp. 991-997

[6] Clifford, M. A., Banovac, F., Levy, E., Cleary, K., Assessment Of Hepatic Motion Secondary To Respiration For Computer Assisted Interventions, Computer Aided Surgery, 7, 2002, pp. 291 - 299

[7] S. Nicolau, A. Garcia, X. Pennec, L. Soler, and N. Ayache, Augmented reality guided radio-frequency tumor ablation, in Computer Animation and Virtual World, 2004,

[8] Kohonen, T., The Basic SOM in "Self-Organizing Maps" „Huang, T. S., Kohonen, T., Schroeder, M. R., (Hrsg.) Springer Verlag, Berlin Heidelberg New York, 1995, 1. Auflage, 77 - 127

[9] J.-Y. $\quad$ Bouguet. $\quad$ Camera $\quad$ Calibration $\quad$ Toolbox for http://www.vision.caltech.edu/bouguetj/calib_doc/, June 2004

[10] S. Umeyama, Least-Squares Estimation of Transformation Parameters Between Two Point Patterns, IEEE Transactions on Pattern Analysis and Machine Intelligence, 13(4), 1991, pp. 376-480.

[11] Balter, J. M., Dawson, L. A., Kazanjian, S., McGinn, C. J., Brock, K. K., Lawrence, T., Ten Haken, R. K., Determination Of Ventilatory Liver Movement Via Radiographic Evaluation Of Diaphragm Position, Int.J.Radiation Oncology Biol.Phys., 51 (2001) 267 - 270

[12] Wong, J. W., Sharpe, M. B., Jaffray, D. A., Kini, V. R., Robertson, J. M., Stromberg, J. S., Martinez, A. A., The Use Of Active Breathing Control (ABC) To Reduce Margin For Breathing Motion, Int.J.Radiation Oncology Biol.Phys., 44 1999, pp. 911 - 919 